\title{
ON THE LATTICE OF CONGRUENCES ON A BAND
}

\author{
G. R. BAIRD* \\ (Received 3 September 1970) \\ Communicated by G. B. Preston
}

Lattices of congruences have been studied by many authors. Hall [4] has shown that the lattice of congruences on a semilattice satisfy a certain chain condition which is a natural extension to arbitrary lattices of the Jordan-Dedekind chain condition for finite lattices.

In this paper we show that this chain condition is also satisfied by the lattice of structure semilattice preserving congruences on any normal band. We in fact show that the lattice of structure semilattice preserving congruences on a normal band satisfies the condition (1) if $y>x \wedge y$ then $x \vee y \succ x$ for any lattice elements $x$ and $y$.

Two examples are included which show that the results given are in a certain sense the best possible. The notation of [1] and [2] is used throughout this paper.

\section{Congruences on regular bands}

We begin by giving the fundamental decomposition theorem for idempotent semigroups. A band $S$ is called rectangular if $x y z=x z$ for all $x, y, z \in S$.

THEOREM 1.1. (McLean [8]). Let $S$ be a band. Then there exists a semilattice $\Gamma$, which is unique up to isomorphism, and a disjoint family of rectangular subbands of $S$ indexed by $\Gamma,\left\{S_{\gamma}: \gamma \in \Gamma\right\}$, such that

(i) $S=\cup\left\{S_{\gamma}: \gamma \in \Gamma\right\}$, and

(ii) $S_{\alpha} S_{\beta} \subseteq S_{\alpha \beta}$ for all $\alpha, \beta \in \Gamma$.

The semilattice $\Gamma$ is the maximal semilattice homomorphic image of $S$. $\Gamma$ is called the structure semilattice of $S$ and $S_{\gamma}$ the $\gamma$-kernel, or simply, a kernel of $S$. We denote the decomposition by the notation:

$$
S \sim \Sigma\left\{S_{\gamma}: \gamma \in \Gamma\right\} .
$$

* This research was carried out at Monash University while the author held a Commonwealth Postgraduate Award. 
The homomorphism of $S$ onto $\Gamma$, which in fact maps $a \in S_{\alpha}$ onto $\alpha$, is called the natural map of $S$ onto $\Gamma$.

Let $\rho$ be a congruence on a band $S \sim \Sigma\left\{S_{\gamma}: \gamma \in \Gamma\right\}$ and suppose the structure semilattice of $S / \rho$ is $\Delta$. Then, since $\Gamma$ is the maximal semilattice homomorphic image of $S$, there is induced a congruence $F$ on $\Gamma$ such that $\Gamma / F \cong \Delta$. We may identify $\Gamma / F$ and $\Delta$. When we wish to indicate explicitly that $\rho$ induces the congruence $F$ on $\Gamma$ we shall write $\rho \rightarrow F$.

LEMMA 1.2. Let $\rho$ be a congruence on $S \sim \Sigma\left\{S_{\gamma}: \gamma \in \Gamma\right\}$ such that $\rho \rightarrow F$. Let $(\alpha, \beta) \in F$ and suppose that $\alpha \geqq \beta$. Then there exist $a \in S_{\alpha}, b \in S_{\beta}$ such that $(a, b) \in \rho$.

Conversely, if $(a, b) \in \rho$ with $a \in S_{\alpha}, b \in S_{\beta}$ then $(\alpha, \beta) \in F$.

Proof. Suppose that $(\alpha, \beta) \in F$. Let $x \in S_{\alpha}$ and $y \in S_{\beta}$. Since $(\alpha, \beta) \in F,(x \rho)$ and $(y \rho)$ are in the same kernel of $S / \rho$ and so

$$
(x \rho)(y \rho)(x \rho)=(x \rho) .
$$

Now $x y x \in S_{\beta}$, since $\alpha \geqq \beta$, and $(x y x) \rho=(x \rho)(y \rho)(x \rho)$. Put $a=x$ and $b=x y x$. Then $a \in S_{\alpha}, b \in S_{\beta}$ and $(a, b) \in \rho$.

The converse is obvious. This completes the proof of Lemma 1.2.

We now determine the congruence $F$ in terms of the congruence $\rho$.

THEOREM 1.3. Let $\rho$ be a congruence on $S \sim \Sigma\left\{S_{\gamma}: \gamma \in \Gamma\right\}$ such that $\rho \rightarrow F$. Then

$$
\begin{gathered}
F=\left\{(\alpha, \beta) \in \Gamma \times \Gamma: \text { there exist } e \in S_{\alpha}, f \in S_{\beta}\right. \text { such that } \\
(e, f) \in \rho\}^{t}
\end{gathered}
$$

REMARK. Let $\sigma$ be a relation on a semigroup $S$. By $\sigma^{t}$ we mean the transitive closure of $\sigma$.

Proof. Put $\sigma=\left\{(\alpha, \beta) \in \Gamma \times \Gamma\right.$ : there exist $e \in S_{\alpha}, f \in S_{\beta}$ such that $\left.(e, f) \in \rho\right\}$. We are required to prove that $F=\sigma^{t}$.

It follows from the second part of Lemma 1.2 that $\sigma^{t} \subseteq F$.

Suppose now that $(\alpha, \beta) \in F$. Then $(\alpha, \alpha \beta) \in F$ and $(\alpha \beta, \beta) \in F$. By Lemma 1.2 there exists $e \in S_{\alpha}, f, g \in S_{\alpha \beta}$, and $h \in S_{\beta}$ such that $(e, f) \in \rho$ and $(g, h) \in \rho$.

Thus $(\alpha, \alpha \beta) \in \sigma$ and $(\alpha \beta, \alpha) \in \sigma$. Hence $(\alpha, \beta) \in \sigma^{t}$ and so $F \subseteq \sigma^{t}$. This completes the proof of Theorem 1.3.

Let $L \sim \Sigma\left\{L_{y}: \gamma \in \Gamma\right\}$ and $R \sim \Sigma\left\{R_{\gamma}: \gamma \in \Gamma\right\}$ be two bands which have the same structure semilattice $\Gamma$. Let $\phi$ and $\psi$ be the natural maps associated with $L$ and $R$ respectively. Then

$$
T=\{(a, b): a \in L, b \in R \text { and } a \phi=b \psi\}
$$


forms a subsemigroup of the direct product $L \times R$. We call $T$ the spined product of $L$ and $R$ with spine $\Gamma$ and write $T=L \bigotimes R(\Gamma)$. Spined products were introduced by Kimura in $[5,7]$.

A band $S$ is called (1) left regular, (2) right regular, or (3) regular if it satisfies respectively the following identities.

(1) $a b a=a b \quad$ for all $a, b \in S$;

(2) $a b a=b a$ for all $a, b \in S$;

(3) $a b a c a=a b c a$ for all $a, b, c \in S$.

THEOREM 1.4 (Kimura [7]). A band $T$ is regular if and only if it is the spined product $L \backslash X \mid R(\Gamma)$ of a left regular band $L$ and a right regular band $R$.

Let $T=L \backslash \backslash \mid R(\Gamma)$ be a regular band. Further, let $\rho_{L}\left(\rho_{R}\right)$ be a congruence on $L(R)$ such that $\rho_{L} \rightarrow F\left(\rho_{R} \rightarrow F\right)$ for some congruence $F$ on $\Gamma$. Put

$$
\pi=\left\{((a, b),(c, d)) \in T \times T:(a, c) \in \rho_{L},(b, d) \in \rho_{R}\right\} .
$$

It is straightforward to check that $\pi$ is a congruence on $T$. We write $\pi=\rho_{L} \backslash \mid \rho_{R}$ and say that $\pi$ is the spined product of $\rho_{L}$ and $\rho_{R}$.

THEOREM 1.5. (This is a restatement of corollary 2 of [7].) Let $S$ and $T$ be regular bands and suppose that $S=A|\bigotimes| B(\Gamma)$ and $T=C \backslash \mid D(\Delta)$, where $A, C$ are left regular and $B, D$ are right regular. Further, suppose that $A \sim \Sigma\left\{A_{\gamma}: \gamma \in \Gamma\right\}, B \sim \Sigma\left\{B_{\gamma}: \gamma \in \Gamma\right\}, C \sim \Sigma\left\{C_{\gamma}: \gamma \in \Delta\right\}$ and $D \sim \Sigma\left\{D_{\gamma}: \gamma \in \Delta\right\}$.

Let $k: S \rightarrow T$ be a homomorphism, then there exists a homomorphism $h: \Gamma \rightarrow \Delta$ and homomorphisms $f: A \rightarrow C$ and $g: B \rightarrow D$ satisfying $(a, b) k=(a f, b g)$ and (2) $p h=f r$ and $q h=g s$. That is, the diagram

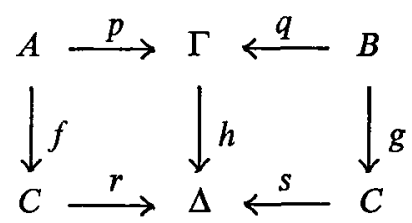

is commutative, where $p, q, r$ and s are the natural homomorphisms.

THEOREM 1.6. Let $\pi$ be a congruence on a regular band $T=L|\backslash| R(\Gamma)$, where $L, R$ are left and right regular bands respectively. Then there exist congruences $\rho_{L}, \rho_{R}$ on Land $R$ respectively such that $\pi=\rho_{L} \bowtie \mid \rho_{R}$.

Proof. Now $T / \pi$ is a regular band and so $T / \pi=L^{\prime} \backslash \backslash \mid R^{\prime}(\Gamma / F)$, where $L^{\prime}$ is a left regular band, $R^{\prime}$ is a right regular band, and $\pi \rightarrow F$. By theorem 1.5 there exist maps $f, g$ from $L$ to $L^{\prime}$ and $R$ to $R^{\prime}$ respectively such that $(a, b) \pi^{\natural}=(a f, b g)$. Put $\rho_{L}=f \circ f^{-1}$ and $\rho_{R}=g \circ g^{-1}$. Then $\rho_{L} \rightarrow F, \rho_{R} \rightarrow F$, again using theorem 1.5. Put $\pi^{*}=\rho_{L} \backslash \backslash \mid \rho_{R}$. Then 
Now

$$
((a, b),(c, d)) \in \pi \text { if and only if }(a, b) \pi^{\natural}=(c, d) \pi^{\natural}
$$

Further,

$$
(a, b) \pi^{\natural}=(c, d) \pi^{\natural} \text { if and only if }(a f, b g)=(c f, d g) .
$$

$$
(a f, b g)=(c f, d g) \text { if and only if }((a, b),(c, d)) \in \pi^{*} .
$$

We conclude that $\pi=\rho_{L} \backslash \mid \rho_{R}$. This completes the proof of theorem 1.6.

Hereafter for a regular band $T$ if we simply write $T=L \backslash X \mid R(\Gamma)$ without any explanation, then $L$ and $R$ will always denote a left regular band and a right regular band respectively. Further for a congruence $\pi$ on a regular band $T=L \backslash \backslash R(\Gamma)$ if we simply write $\pi=\rho \backslash \backslash \mid \sigma$, then $\rho, \sigma$ will mean congruences on $L, R$ respectively.

LEMMA 1.7. Let $\pi_{1}, \pi_{2}$ be congruences on a regular band $S=L \backslash \backslash R(\Gamma)$. Suppose that $\pi_{1}=\rho_{1} \backslash \backslash \sigma_{1}$ and $\pi_{2}=\rho_{2} \backslash \mid \sigma_{2}$. Then $\pi_{1} \subseteq \pi_{2}$ if and only if $\rho_{1} \subseteq \rho_{2}$ and $\sigma_{1} \subseteq \sigma_{2}$.

Proof. If $\rho_{1} \subseteq \rho_{2}$ and $\sigma_{1} \subseteq \sigma_{2}$ then by the definition of spined product

$$
\rho_{1} \backslash\left|\sigma_{1} \subseteq \rho_{2}\right| X \mid \sigma_{2} \text {. }
$$

On the other hand assume that $\pi_{1} \subseteq \pi_{2}$. Suppose that

$$
S / \pi_{1}=L_{1} \backslash \mid R_{1}\left(\Gamma / F_{1}\right) \text { and } S / \pi_{2}=L_{2}|\backslash| R_{2}\left(\Gamma / F_{2}\right) \text {, }
$$

where $L_{1}, L_{2}$ are left regular bands, $R_{1}, R_{2}$ are right regular bands, and $\pi_{1} \rightarrow F_{1}$, $\pi_{2} \rightarrow F_{2}$. Let $f_{1}$ and $f_{2}$ be the maps from $L$ to $L_{1}$ and $L$ to $L_{2}$ respectively such that

$$
\rho_{1}=f_{1} \circ f_{1}^{-1} \text { and } \rho_{2}=f_{2} \circ f_{2}^{-1} .
$$

Since $\pi_{1} \subseteq \pi_{2}$ the map $\pi_{2}^{\natural}$ from $S$ to $S / \pi_{2}$ can be factored through $S / \pi_{1}$. That is, there exists a map $\psi_{1}$ from $S / \pi_{1}$ to $S / \pi_{2}$ such that $\pi_{2}^{\natural}=\pi_{1}^{\natural} \psi_{1}$. It follows from theorem 1.5 that there exists a map $\psi_{2}$ from $L_{1}$ to $L_{2}$ such that $f_{2}=f_{1} \psi_{2}$. Hence

$$
f_{1} \circ f_{1}^{-1} \subseteq f_{2} \circ f_{2}^{-1},
$$

that is, $\rho_{1} \subseteq \rho_{2}$. Similarly, we prove that $\sigma_{1} \subseteq \sigma_{2}$. This completes the proof of lemma 1.7.

THEOREM 1.8. Let $\pi_{1}=\lambda_{1} \bigotimes \mid \sigma_{1}$ and $\pi_{2}=\lambda_{2}|\backslash| \sigma_{2}$ be congruences on a regular band. Then
(1) $\pi_{1} \vee \pi_{2}=\lambda_{1} \vee \lambda_{2} \backslash \mid \sigma_{1} \vee \sigma_{2}$, and
(2) $\pi_{1} \wedge \pi_{2}=\lambda_{1} \wedge \lambda_{2} \chi_{\mid} \sigma_{1} \wedge \sigma_{2}$.

Theorem 1.8 follows from lemma 1.7 using routine lattice theoretic arguments. We omit the details. 


\section{Some definitions and known results}

THEOREM 2.1. (This is easy to show and is largely contained in section 3 of [9].) Let $S$ be any set and consider the set of equivalences on $S$, ordered under set inclusion. Let $\sigma$ be any equivalence on $S$. Then there is a natural order-preserving one-to-one correspondence (described below) between the set of equivalences on $S$ which contain $\dot{\sigma}$ and the set of all equivalences on the set $S / \sigma$. For any equivalence $\gamma$ on $S$ containing $\sigma$, the corresponding equivalence on $S / \sigma$ is

$$
\gamma / \sigma=\{(x \sigma, y \sigma) \in S / \sigma \times S / \sigma:(x, y) \in \gamma\} .
$$

If $S$ is a semigroup and $\sigma$ is a congruence on $S$ then an equivalence $\gamma$ on $S$ containing $\sigma$ is a congruence on $S$ if and only if $\gamma / \sigma$ is a congruence on the semigroup $S / \sigma$.

The following definitions and results are contained in [3], except for the definition of upper semimodularity, which is merely called condition (7) in [3].

Let $L$ be a lattice. For $a, b \in L, a>b$ means that $a \geqq b$ and that $a \neq b$, and $a \succ b$ (a covers $b$ ) means that $a>b$ and there is no element $x \in L$ such that $a>x>b$. For a lattice $L$ with a zero element 0 , the atoms of $L$ are those elements which cover 0 .

Definition 2.2. (This is a restatement of definition 1, page 85 [3].) A lattice $L$ is semimodular when for any elements $x, y, z \in L$ if

$$
y \wedge z<x<z<x \vee y
$$

then there exists an element $t \in L$ such that

$$
y \wedge z<t \leqq y \text { and } x=(x \vee t) \wedge z
$$

Definition 2.3. (This is a restatement of condition (7), page 92 [3].). A lattice $L$ is upper-semimodular when for any elements $x, y \in L$, if $x>x \wedge y$ and $y>x \wedge y$ then $x \vee y>x$ and $x \vee y>y$.

Theorem 2.4. (This follows from lemma 1 and theorem 1 on page 265 [3].). The lattice $\xi(S)$ of all equivalences on a set $S$ is semimodular.

THEOREM 2.5. (This is a restatement of property 2, page 90 [3].). Any semimodular lattice satisfies the following condition:

(1) if $y \succ x \wedge y$ then $x \vee y \succ x$, for any lattice elements $x$ and $y$.

THEOREM 2.6. (This is a restatement of the last paragraph of page 92 [3].) Any lattice satisfying condition (1) is upper-semimodular.

TheOREM 2.7. (This is a restatement of theorem 1, page 88 [3].) Let L be any lattice satisfying condition (1), and let $a, b$ be elements of L such that $a<b$. 
If there is a maximal chain of elements from a to $b$ which is of finite length, then any maximal chain of elements from $a$ to $b$ is finite and of the same length.

Let $S$ be a semigroup and let $\Lambda(S)$ be the lattice of all congruences on $S$. Suppose now that $S$ is a band and that

$$
S \sim \Sigma\left\{S_{\gamma}: \gamma \in \Gamma\right\} .
$$

We define

$$
\Lambda_{i}(S)=\left\{\sigma \in \Lambda(S):(a, b) \in \sigma \text { implies there exists } \alpha \in \Gamma \text { such that } a, b \in S_{\alpha}\right\} .
$$

It is clear that $\rho \in \Lambda_{i}(S)$ if and only if $\phi \circ \phi^{-1} \supseteq \rho$, where $\phi$ is the natural map of $S$ upon $\Gamma$. Thus $\Lambda_{i}(S)$ is a sublattice of $\Lambda(S)$. In fact $\Lambda_{i}(S)$ is a complete sublattice of $\Lambda(S)$.

Evidently if $\rho \in \Lambda_{i}(S)$ then the structure semilattice of $S / \rho$ is isomorphic to $\Gamma$ and hence we call $\Lambda_{i}(S)$ the sublattice of structure semilattice preserving congruences on $S$.

The following example due to Hall [4] shows that the lattice of congruences on a regular band is not upper-semimodular. In fact we show that the lattice of structure semilattice preserving congruences on a right regular band is not upper-semimodular.

Put $S=\{a, b, e, f, g, h\}$ with multiplication given by the table

\begin{tabular}{c|cccccc} 
& $a$ & $b$ & $e$ & $f$ & $g$ & $h$ \\
\hline$a$ & $a$ & $b$ & $e$ & $f$ & $g$ & $h$ \\
$b$ & $a \mathbf{3}$ & $b$ & $e$ & $-f$ & $g$ & $h$ \\
$e$ & $e$ & $g$ & $e$ & $f$ & $g$ & $h$ \\
$f$ & $f$ & $h$ & $e$ & $f$ & $g$ & $h$ \\
$g$ & $e$ & $g$ & $e$ & $f$ & $g$ & $h$ \\
$h$ & $f$ & $h$ & $e$ & $f$ & $g$ & $h$
\end{tabular}

It is routine to check that $S$ is a right regular idempotent semigroup. Consider the relations

$$
\begin{aligned}
\rho_{1} & =\{(e, f),(f, e),(\mathrm{g}, h),(h, g)\} \cup i_{s} \\
\rho_{2} & =\{(e, g),(g, e)\} \cup i_{s} \text { and } \\
\sigma & =\{(e, g),(g, e),(f, h),(h, f)\} \cup i_{\mathrm{s}}
\end{aligned}
$$


It is easily checked that $\rho_{1}, \rho_{2}$ and $\sigma$ are contained in $\Lambda_{i}(S)$. Further, $\rho_{1} \succ i_{s}$ and $\rho_{2} \succ i_{s}$. Now

$$
\begin{aligned}
\rho_{1} \vee \rho_{2}= & \{(e, f),(e, g),(e, h),(f, e),(g, e),(h, e), \\
& (f, g),(f, h),(g, f),(h, f),(g, h),(h, g)\} \cup i_{s} .
\end{aligned}
$$

Thus $\rho_{2}<\sigma<\rho_{1} \vee \rho_{2}$. Hence $\Lambda_{i}(S)$ is not upper-semimodular.

In view of the above example we now focus our attention on a smaller class of bands.

\section{Congruences on normal bands}

A band $S$ is called 1. left normal, 2. right normal, or 3. normal if it satisfies respectively the following identities:

1. $a b c=a c b$ for all $a, b, c \in S$;

2. $a b c=b a c$ for all $a, b, c \in S$;

3. $a b c d=a c b d$ for all $a, b, c, d \in S$.

THEOREM 3.1 (Kimura and Yamada [6]) $A$ band is normal if and only if it is a spined product of a left normal band and a right normal band.

We remark that a left normal band is left regular, a right normal band is right regular and a normal band is regular. Furthermore, a normal left regular band is left normal and a normal right regular band is right normal. Hence theorem 3.1 is a corollary to theorem 1.4 .

By the above remark any congruence $\pi$ on a normal band $S=L \backslash \backslash R(\Gamma)$, can be written as a spined product $\pi=\rho \bigotimes \mid$, where $\rho$ is a congruence on the left normal band $L$ and $\sigma$ is a congruence on the right normal band $R$.

Let $\rho \in \Lambda_{i}(S)$ and suppose that $(e, f) \in \rho, e \neq f$. Clearly there exist $\alpha \in \Gamma$ such that $e, f \in S_{\alpha}$. Generalizing Hall's argument in [4] put

$$
\sigma=i_{s} \cup\left\{(x, y) \in S \times S:(x, y) \in \rho, x \in S_{\beta}, \beta \leqq \alpha\right\} .
$$

It is straightforward to check that $\sigma$ is a congruence and that $i_{s}<\sigma \leqq \rho$. Suppose now that $\rho$ covers $i_{s}$. Then $\sigma=\rho$ and moreover,

$$
\rho=i_{s} \cup\left[\rho \cap S_{\alpha} \times S_{\alpha}\right]
$$

for suppose that $(g, h) \in \rho, g \neq h, g \in S_{\mu}$, and $\mu<\alpha$. Then

$$
\sigma^{\prime}=i_{s} \cup\left\{(x, y) \in S \times S:(x, y) \in \rho, x \in S_{v}, v<\alpha\right\}
$$

is a congruence such that $i_{s}<\sigma^{\prime}<\rho$, a contradiction.

THEOREM 3.2. Let $S \sim \sum\left\{S_{\gamma}: \gamma \in \Gamma\right\}$ be a left normal band, $\rho$ any atom of $\Lambda_{i}(S)$. Then there exists $e, f \in S_{\alpha}$, such that 
(i) $\rho=\{(e, f),(f, e)\} \cup i_{s}$

(ii) $e x=f x$ for all $x \in S_{\beta}, \beta<\alpha$.

Conversely, given any pair $e, f \in S_{\alpha}$ such that (ii) holds, the relation $\rho$ defined by (i) is a congruence and hence an atom of $\Lambda_{i}(S)$.

Proof. We begin by proving the converse. It is easily checked, by using theorem 1 in [6], that $\rho$ is a congruence. The converse is now obvious.

Let now $\rho$ be an atom of $\Lambda_{i}(S)$ and suppose $(e, f) \in \rho, e \neq f$. Clearly $e, f \in S_{\alpha}$ for some $\alpha \in \Gamma$. Now (ex,fx) $\rho$ for all $x \in S$ since $\rho$ is a congruence and so (ii) holds using (A). Put

$$
\sigma=\{(e, f),(f, e)\} \cup i_{s} .
$$

Then $\sigma$ is an atom (the converse) and $i_{s}<\sigma \leqq \rho, \sigma=\rho$ and the proof of theorem 3.2 is complete.

THEOREM 3.3. Let $S$ be a left normal band. For any two congruences $\rho, \sigma \in \Lambda_{i}(S) \rho \succ \sigma$ in the lattice $\Lambda_{i}(S)$ if and only if $\rho \succ \sigma$ in the lattice $\xi(S)$.

Proof. The "if" statement is obvious.

Suppose that $\rho \succ \sigma$ in the lattice $\Lambda_{i}(S)$. Then $\rho / \sigma \in \Lambda_{i}(S / \sigma)$ (by theorem 2.1) and since the one-to-one correspondence of theorem 2.1 is order preserving, $\rho / \sigma$ is an atom of $\Lambda_{i}(S / \sigma)$. But $S / \sigma$ is also a left normal band, whence by theorem $3.2 \rho / \sigma$ is an atom of $\xi(S / \sigma)$. Using theorem 2.1 again we obtain that $\rho \succ \sigma$ in the lattice $\xi(S)$. This completes the proof of theorem 3.3.

THEOREM 3.4. Let $S$ be a left normal band. Then the lattice $\Lambda_{i}(S)$ satisfies the condition (1).

Proof. Recall that $\Lambda(S)$ is a sublattice of $\xi(S)$. Further, $\Lambda_{i}(S)$ is a sublattice of $\Lambda(S)$ and so $\Lambda_{i}(S)$ is a sublattice of $\xi(S)$. But $\xi(S)$ satisfies condition (1) by theorems 2.4 and 2.5. Theorem 3.4 now follows from theorem 3.3. This completes the proof of theorem 3.4.

LEMMA 3.5. Let $S=L \bigotimes R(\Gamma)$ be a normal band. Let $\pi$ be an atom of $\Lambda_{i}(S)$. Then $\pi=\rho \bigvee i_{R}$, where $\rho$ is an atom of $\Lambda_{i}(L)$, or $\pi=i_{L} \backslash \mid \rho$, where $\rho$ is an atom of $\Lambda_{i}(R)$.

Lemma 3.5 follows immediately from lemma 1.7 and the definition of an atom.

LEMMA 3.6. Let $S=L \bigotimes R(\Gamma)$ be a normal band. Let $\pi_{1}=\rho_{1}|\backslash| \sigma_{1}$ $\pi_{2}=\rho_{2} \backslash \backslash \sigma_{2} \in \Lambda_{i}(S)$. Then $\pi_{1}>\pi_{2}$ if and only if

(i) $\rho_{1} \succ \rho_{2}$ and $\sigma_{1}=\sigma_{2}$, or

(ii) $\rho_{1}=\rho_{2}$ and $\sigma_{1} \succ \sigma_{2}$. 
Proof. We observe that $\pi_{1} / \pi_{2}$ is an atom of $S / \pi_{2}$ and that $\pi_{1} / \pi_{2}=\rho_{1} / \rho_{2}$ $凶 \sigma_{1} / \sigma_{2}$. Lemma 3.6 now follows from lemma 3.5 and theorem 2.1. This completes the proof of lemma 3.6.

THEOREM 3.7. Let $S=L \backslash \backslash R(\Gamma)$ be a normal band. Then the lattice $\Lambda_{i}(S)$ satisfies condition (1).

Proof. Let $\pi_{1}=\rho_{1} \backslash\left|\sigma_{1}, \pi_{2}=\rho_{2} \backslash \backslash\right| \sigma_{2} \in \Lambda_{i}(S)$ and suppose that $\pi_{1} \succ \pi_{1} \wedge \pi_{2}$. Now

$$
\pi_{1} \wedge \pi_{2}=\rho_{1} \wedge \rho_{2} \backslash X \mid \sigma_{1} \wedge \sigma_{2}
$$

Hence

$$
\rho_{1} \succ \rho_{1} \wedge \rho_{2} \text { and } \sigma_{1}=\sigma_{1} \wedge \sigma_{2}
$$

or

$$
\rho_{1}=\rho_{1} \wedge \sigma_{2} \text { and } \sigma_{1} \succ \sigma_{1} \wedge \sigma_{2}
$$

by lemma 3.6. Suppose that $\rho_{1}>\rho_{1} \wedge \rho_{2}$ and $\sigma_{1}=\sigma_{1} \wedge \sigma_{2}$. Then $\sigma_{1} \vee \sigma_{2}=\sigma_{2}$. Hence $\pi_{1} \vee \pi_{2}=\rho_{1} \vee \rho_{2} \ \mid \sigma_{2}$. Further, $\rho_{1} \vee \rho_{2} \succ \rho_{2}$ since $L$ is a left normal band and theorem 3.4. Hence $\pi_{1} \vee \pi_{2} \succ \pi_{2}$.

We treat the other case similarly using the left-right dual of theorem 3.4. This completes the proof of theorem 3.7.

CoROLlary 3.8. Let $S$ be a normal band. If $\rho, \sigma \in \Lambda_{i}(S)$ such that $\sigma \leqq \rho$ and there is a maximal chain of congruences from $\sigma$ to $\rho$ which is of finite length, then any maximal chain of congruences from $\dot{\sigma}$ to $\rho$ is finite and of the same length.

COROLlaRY 3.9. The lattice $\Lambda_{i}(S)$ of structure semilattice preserving congruences on a normal band is upper-semimodular.

Corollaries 3.8 and 3.9 follow immediately from theorems 3.7, 2.7 and 2.6.

We conclude this paper with an example showing that corollary 3.9 does not extend to the full lattice. Let $S=\{a, b, x, y\}$ with multiplication table given by

\begin{tabular}{c|cccc} 
& $a$ & $b$ & $x$ & $y$ \\
\hline$a$ & $a$ & $a$ & $x$ & $x$ \\
$b$ & $b$ & $b$ & $y$ & $y$ \\
$x$ & $x$ & $x$ & $x$ & $x$ \\
$y$ & $y$ & $y$ & $y$ & $y$
\end{tabular}


It is routine to check that $S$ is a left normal band. The lattice $\Lambda(S)$ is isomorphic to the lattice given diagrammatically below.

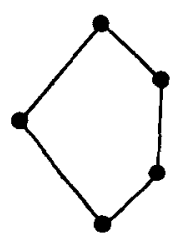

Hence $\Lambda(S)$ is not upper-semimodular.

\section{References}

[1] A. H. Clifford and G. B. Preston, The algebraic theory of semigroups, Volume I (Maths. Surveys, Number 7, Amer. Math. Soc. 1961).

[2] A. H. Clifford and G. B. Preston, The algebraic theory of semigroups, Volume II (Maths. Surveys, Number 7, Amer. Math. Soc. 1967).

[3] M. L. Dubreil-Jacotin, L. Lesieur and R. Croisot, Lecons sur la théorie des treillis des structures algébriques ordonnées et des treillis géometriques, (Paris, Gauthier-Villars, Editeurimprimeur-libraire, 1953).

[4] T. Hall, 'On the lattice of congruences on a semilattice', J. Aust. Math. Soc. (to appear).

[5] N. Kimura, 'Note on idempotent semigroups I', Proc. Japan Acad. 33 (1957), 642-645.

[6] N. Kimura and M. Yamada, 'Note on idempotent semigroups II', Proc. Japan Acad. 34 (1958), 110-112.

[7] N. Kimura, 'The structure of idempotent semigroups (1)', Pacific J. Math. 8 (1957), 257-275.

[8] D. McLean, 'Idempotent semigroups', Amer. Math. Monthly 61 (1954), 110-113.

[9] N. R. Reilly and H. E. Scheiblich, 'Congruences on regular semigroups', Pacific J. Math. 23 (1967), 349-360. 